JOURNAL OF THE

AMERICAN MATHEMATICAL SOCIETY

Volume 20, Number 4, October 2007, Pages 969-988

S 0894-0347(07)00568-1

Article electronically published on May 31, 2007

\title{
EDGE COLORING MODELS AND REFLECTION POSITIVITY
}

\author{
BALÁZS SZEGEDY
}

\section{INTRODUCTION}

The motivation of this paper comes from statistical physics as well as from combinatorics and topology. The general setup in statistical mechanics can be outlined as follows. Let $G$ be a graph and let $\mathcal{C}$ be a finite set of "states" or "colors". We think of $G$ as a crystal in which either the edges or the vertices are regarded as "sites" which can have states from $\mathcal{C}$. In the first case we speak about edge coloring models and in the second case about vertex coloring models. A configuration of the whole system is a function which associates a state with each site. The states are interacting with each other at the vertices in edge coloring models and along edges in vertex coloring models. A weight is associated with each such interaction which is a real (or complex) number depending on the interacting states (in vertex coloring models there are additional weights associated with the states). A concrete model is usually given by these numbers. The partition function can be interpreted as a graph parameter which is computed by summing the products of the weights over all possible configurations of the system represented by $G$. It proves to be useful to extend this graph parameter linearly to the vector space of formal linear combinations of graphs. The elements of this vector space are called quantum graphs. Quantum graphs that can be obtained by gluing together a quantum graph with its reflected version (using the distributive law) are called reflection symmetric. However there are two different reasonable definitions of gluing. In the first one we glue along unfinished edges and in the second one along vertices. Correspondingly we get the notions of edge reflection symmetric and vertex reflection symmetric quantum graphs. A graph parameter is called edge reflection positive (resp. vertex reflection positive) if it takes nonnegative values on edge-reflection symmetric (resp. vertex reflection symmetric) quantum graphs. It is a simple fact that the partition function in edge coloring models is edge reflection positive and is vertex reflection positive in vertex coloring models. A surprising result proved by M. H. Freedman, L. Lovász and A. Schrijver (see [4]) says that vertex reflection positivity is almost enough to characterize the partition functions of vertex coloring models. The extra condition that they need is that the ranks of certain matrices (which describe the gluing operation and are called connection matrices) are growing at most exponentially. They conjectured that similar characterization can be given for edge reflection positive graph parameters. The main result of this paper (theorem 2.2) is the proof of this conjecture in a strong version where we replace the condition on the rank growth by a weak and

Received by the editors May 2, 2005.

2000 Mathematics Subject Classification. Primary 05C99; Secondary $82 B 99$.

(C)2007 American Mathematical Society Reverts to public domain 28 years from publication 
natural condition, namely that the graph parameter is multiplicative for taking a disjoint union of graphs.

The major difficulty of the proof is coming from a fact which is interesting in its own right: In contrast with vertex coloring models, partition functions of edge coloring models don't determine the weights. There is an action of the orthogonal group on different edge coloring models, which leaves the corresponding partition function invariant. This phenomenon explains why it is difficult to reconstruct an edge coloring model from its partition function. In contrast with vertex coloring models we are searching for an orbit of the orthogonal group rather than one specific object. Our main tools to handle this difficulty are commutative algebra and the theory of invariants of the orthogonal group.

A topological version of the above described reflection symmetry and reflection positivity arises in topological quantum field theory (see [1] and [3]) where the gluing operation is defined on the formal linear space of manifolds with a fixed boundary.

We should also emphasize that the subject has a close connection to pure combinatorics. The partition function of a vertex coloring model can be interpreted as the number of graph homomorphisms into a fixed graph. This shows that the number of proper colorings and many related important graph parameters are coming from vertex coloring models (see [4). In many other cases where we count certain structures in a graph (perfect matchings, fully packed loop configurations, etc.) it turns out that this number is the value of the partition function of an edge coloring model. The orthogonal invariance of edge coloring models generates interesting equations between such numbers (a simple example is shown in section 3.2). Another peculiar fact that we show is that vertex coloring models can be represented by complex valued edge coloring models such that the values of the two partition functions are identical. In some special cases the representing edge coloring model is also real valued and in this case the corresponding graph parameter is both vertex and edge reflection positive. We show that the Ising model is such an example. Finally we mention that a version of vertex coloring models with an infinite number of states is worked out and characterized in [7. In such a model the states are elements of a measure space on which the weights are given by a measurable function. From the combinatorial point of view, these vertex coloring models can be regarded as limits of sequences of finite graphs and such objects are relevant to extremal combinatorics. In section 3.1 we point out that some of these infinite models can be represented by edge coloring models with finitely many states.

\section{EdGE COLORING MODELS AND REFLECTION POSITIVITY}

2.1. Circles and quantum graphs. Throughout the paper it will be convenient to extend the concept of graphs by introducing edges that are not incident to any vertex. We call such edges circle edges and picture them as topological circles. Formally, a circle is an element of the edge set which has no endpoints. Let $\mathcal{G}$ denote the set of isomorphism classes of graphs, in which loops, circles and multiple edges are allowed. We denote by $\emptyset \in \mathcal{G}$ the empty graph whose vertex and edge sets are both empty. If $G_{1}, G_{2} \in \mathcal{G}$ are two graphs then their disjoint union $G_{1} \cup G_{2}$ is defined to be a graph whose vertex set and edge set is the disjoint union of those of $G_{1}$ and $G_{2}$. Every element of $\mathcal{G}$ is the disjoint union of an ordinary (circle free) graph and a finite number of circles. 
Let $R$ be an arbitrary commutative ring with 1 . An $R$-valued graph parameter is a map $f: \mathcal{G} \rightarrow R$. We say that $f$ is multiplicative if $f\left(G_{1} \cup G_{2}\right)=f\left(G_{1}\right) f\left(G_{2}\right)$ for any two graphs $G_{1}, G_{2} \in \mathcal{G}$ and $f(\emptyset)=1$.

Let $F$ be a field and let $\mathcal{Q}(F)$ denote the vectorspace of finite $F$-linear combinations of elements of $\mathcal{G}$. The elements of $\mathcal{Q}(F)$ are called quantum graphs. The operation of taking disjoint union can be extended to quantum graphs by using the distributive law. It is easy to see that $\mathcal{Q}(F)$ becomes an $F$-algebra with 1 if we introduce disjoint union as multiplication. If the ring $R$ is an $F$-algebra, then any multiplicative graph parameter $f: \mathcal{G} \rightarrow R$ extends uniquely to an algebra homomorphism $f: \mathcal{Q}(F) \rightarrow R$. As a consequence we have that the image of $\mathcal{Q}(F)$ is a subalgebra of $R$.

The usual setting in this paper is that $F$ is the field of real numbers and $R$ is either the field of real numbers or a polynomial ring over the reals. For this reason we use the shorthand notation $\mathcal{Q}$ instead of $\mathcal{Q}(\mathbb{R})$.

2.2. Edge reflection positivity. In this section we will need the notion of graphs with outgoing (open) edges. An outgoing edge can be pictured as an edge which goes out from the graph but is not finished. It can also happen that such an edge goes out in both directions and so it is not incident to any of the vertices. However the behavior of these edges is different from circles because we want to maintain the possibility of finishing them. To define this concept precisely we need to introduce the set of "open ends" $O(G)$ of a graph $G$. A graph $G$ with outgoing edges is a triple $(V(G), E(G), O(G))$, where $(O(G) \cup V(G), E(G))$ is a graph in $\mathcal{G}$ with the property that the degrees of open ends are exactly 1.

We define $\mathcal{G}_{k}$ to be the set of all graphs with exactly $k$ open ends which are labeled by the numbers $1,2, \ldots, k$. There is a natural operation

$$
g: \mathcal{G}_{k} \times \mathcal{G}_{k} \rightarrow \mathcal{G}
$$

which is called gluing and defined in the following way. Let $G_{1}$ and $G_{2}$ be two graphs in $\mathcal{G}_{k}$. Let us take the disjoint union of them and identify their open ends which have the same label. This way we obtain a graph in $\mathcal{G}$ in which there are $k$ labeled vertices of degree 2. Finally we eliminate these vertices (and their two incident edges) by introducing a new edge which connects their two neighbors directly. It is easy to see that the resulting graph does not depend on the order in which we eliminate the labeled vertices. Note also that the resulting graph may contain circles even if the original two graphs did not have any. This explains the importance of circles. The notation of gluing is also defined for graphs with no outgoing edges, but in this case gluing is the same as taking disjoint union.

Let $\mathcal{Q}_{k}$ denote the vectorspace of formal $\mathbb{R}$-linear combinations of elements of $\mathcal{G}_{k}$. Now the gluing operation extends uniquely to a symmetric bilinear form

$$
g: \mathcal{Q}_{k} \times \mathcal{Q}_{k} \rightarrow \mathcal{Q} .
$$

We say that a quantum graph $Q \in \mathcal{Q}$ is edge reflection symmetric if $Q=g(H, H)$ for some quantum graph $H \in \mathcal{Q}_{k}$ with $k \geq 0$. A graph parameter $f: \mathcal{G} \rightarrow \mathbb{R}$ is called edge reflection positive if its linear extension $f: \mathcal{Q} \rightarrow \mathbb{R}$ takes nonnegative values on all edge reflection symmetric quantum graphs. In other words $f$ is edge reflection positive if and only if the bilinear forms

$$
f \circ g: \mathcal{Q}_{k} \times \mathcal{Q}_{k} \rightarrow \mathbb{R}
$$


are positive semi-definite for all $k \geq 0$. One can write up the matrices of these scalar products in the natural basis $\mathcal{G}_{k}$ and obtain the so-called connection matrices $M(k, f)$. These are infinite matrices whose rows and columns are indexed by the elements of $\mathcal{G}_{k}$ and the entry in the intersection of the row corresponding to $G_{1}$ and the column corresponding to $G_{2}$ is $f\left(g\left(G_{1}, G_{2}\right)\right)$.

2.3. Edge coloring models and the characterization theorem. Let $R$ be a commutative $\mathbb{R}$-algebra with 1 . (Usually $R=\mathbb{R}$ or a polynomial ring over $\mathbb{R}$.) Let $\mathcal{C}=\left\{c_{1}, c_{2}, \ldots, c_{d}\right\}$ be a finite set of size $d$ whose elements will be referred to as colors. An $R$-valued edge coloring model is given by a function $t: \mathbb{N}^{d} \rightarrow R$ where 0 is considered to be a natural number. For every edge coloring model we are going to define an associated graph parameter $t: \mathcal{G} \rightarrow R$. Let $v \in V(G)$ be a vertex and let $\psi: E(G) \rightarrow \mathcal{C}$ be a coloring of the edge set of a graph $G$. We denote by $v_{\psi} \in \mathbb{N}^{d}$ the vector whose $i$-th coordinate is the number of edges with color $c_{i}$ incident to vertex $v$. It is important that loop edges are counted twice. Now we define $t_{\psi}(G)$ by

$$
t_{\psi}(G)=\prod_{v \in V(G)} t\left(v_{\psi}\right)
$$

and $t(G)$ by

$$
t(G)=\sum_{\psi: E(G) \rightarrow \mathcal{C}} t_{\psi}(G) .
$$

It is clear that $t$ is a multiplicative graph parameter if we define the empty product to be 1 and moreover the value of $t$ on a single circle is the number of colors (which is $d$ ).

Let $k \geq 0$ be a natural number and let $G \in \mathcal{G}_{k}$ be a graph with $k$ labeled outgoing edges. We say that a coloring $\psi: E(G) \rightarrow \mathcal{C}$ is an extension of a coloring $\chi: O(G) \rightarrow \mathcal{C}$ of the open ends if each open end $o \in O(G)$ has the same color as the unique edge incident to $o$. We denote this relation by $\psi>\chi$. For a coloring $\chi: O(G) \rightarrow \mathcal{C}$ we introduce $t_{\chi}(G)$ by

$$
t_{\chi}(G)=\sum_{\psi: E(G) \rightarrow \mathcal{C}, \psi>\chi} t_{\psi}(G) .
$$

Now let $G_{1}$ and $G_{2}$ be two graphs in $\mathcal{G}_{k}$. Since the open ends in both $G_{1}$ and $G_{2}$ are labeled by numbers $1,2, \ldots, k$ we can say, by abusing the notation, that any coloring $\chi:\{1,2, \ldots, k\} \rightarrow \mathcal{C}$ is also a coloring of $O\left(G_{1}\right)$ and $O\left(G_{2}\right)$. Assume that $\psi_{1}>\chi$ in $G_{1}$ and $\psi_{2}>\chi$ in $G_{2}$ for the same coloring $\chi$. Then there is a coloring $\psi=g\left(\psi_{1}, \psi_{2}\right)$ of the edges of $G=g\left(G_{1}, G_{2}\right) \in \mathcal{G}$ which is obtained by gluing together $\psi_{1}$ and $\psi_{2}$. This coloring has the property that $v_{\psi}=v_{\psi_{1}}$ if $v \in V\left(G_{1}\right)$ and $v_{\psi}=v_{\psi_{2}}$ if $v \in V\left(G_{2}\right)$. It follows that

$$
t_{\psi}(G)=t_{\psi_{1}}\left(G_{1}\right) t_{\psi_{2}}\left(G_{2}\right)
$$

and that

$$
t(G)=\sum_{\chi:\{1,2, \ldots, k\} \rightarrow \mathcal{C}} t_{\chi}\left(G_{1}\right) t_{\chi}\left(G_{2}\right) .
$$

It is clear that the previous equality also holds for $G_{1}, G_{2} \in \mathcal{Q}_{k}$ and $G=$ $g\left(G_{1}, G_{2}\right) \in \mathcal{Q}$ if we extend the invariants $t$ and $t_{\chi}$ linearly to quantum graphs from $\mathcal{Q}$ and $\mathcal{Q}_{k}$. As a consequence we get that real valued edge coloring models 
give rise to edge reflection positive graph parameters:

Proposition 2.1. Let $t: \mathbb{N}^{d} \rightarrow \mathbb{R}$ be a real valued edge coloring model. Then the graph parameter $t: \mathcal{G} \rightarrow \mathbb{R}$ is edge reflection positive.

Proof. Let $k \geq 0$ ba a natural number and $Q=g(H, H)$ for some $H \in \mathcal{G}_{k}$. Using equation (11) we have that

$$
t(Q)=\sum_{\chi:\{1,2, \ldots, k\} \rightarrow \mathcal{C}} t_{\chi}(H)^{2} \geq 0 .
$$

Our main theorem is the converse of the previous statement.

Theorem 2.2. Let $f: \mathcal{G} \rightarrow \mathbb{R}$ be an edge reflection positive and multiplicative graph parameter. Then there is an edge coloring model $t: \mathbb{N}^{d} \rightarrow \mathbb{R}$ such that the corresponding graph parameter equals $f$.

The subsequent section will lead to the proof of this theorem.

2.4. Universal edge coloring models. Let us fix a natural number $d$ and let us introduce algebraically independent variables $x_{v}$ for each vector $v \in \mathbb{N}^{d}$. Let $P_{d}$ denote the polynomial ring $\mathbb{R}\left[\left\{x_{v} \mid v \in \mathbb{N}^{d}\right\}\right]$. The universal edge coloring model $t_{d}$ corresponding to $d$ is a $P_{d}$ valued edge coloring model which is given by the function $t_{d}(v)=x_{v}$. An important property of these models is that real valued edge coloring models $t$ with $d$ colors are in one to one correspondence with homomorphisms $\varrho: P_{d} \rightarrow \mathbb{R}$ where the correspondence is given by the equation $\varrho\left(x_{v}\right)=t(v)$. Note that if $t$ and $\varrho$ correspond to each other, then $t(Q)=\varrho\left(t_{d}(Q)\right)$ for all $Q \in \mathcal{Q}$.

Let us introduce

$$
I_{d}=\left\{t_{d}(Q) \mid Q \in \mathcal{Q}\right\} .
$$

Since $t_{d}$ is multiplicative we have that $I_{d}$ is a subring of $P_{d}$. We will later prove that $I_{d}$ is the set of all elements in $P_{d}$ which are invariant under a certain "natural" action of the orthogonal group $O_{d}(\mathbb{R})$.

2.5. Action of the orthogonal group on edge coloring models. Let $d$ be a natural number and let $V$ be the vectorspace consisting of the formal $\mathbb{R}$-linear combinations of the colors $c_{1}, c_{2}, \ldots, c_{d}$. We say that $V$ is the color space and the elements of $V$ will be called quantum colors. The space $V$ is endowed with a euclidean scalar product for which $c_{1}, c_{2}, \ldots, c_{d}$ is an orthonormal basis. Let us fix an edge coloring model $t: \mathbb{N}^{d} \rightarrow R$. For every natural number $n$ we define a symmetric $n$-linear form $l_{n}$ on $V$ by

$$
l_{n}\left(c_{i_{1}}, c_{i_{2}}, \ldots, c_{i_{n}}\right)=t\left(m_{1}, m_{2}, \ldots, m_{d}\right)
$$

where $m_{i}$ denotes the number of occurrence of the color $c_{i}$ on the list $c_{i_{1}}, c_{i_{2}}, \ldots, c_{i_{n}}$.

Let $\alpha$ be an orthogonal transformation of $V$. We denote by $u^{\alpha}$ the image of a vector $u \in V$ under the action of $\alpha$. We define a new edge coloring model $t^{\alpha}$ by

$$
t^{\alpha}\left(i_{1}, i_{2}, \ldots, i_{d}\right)=l_{n}\left(c_{j_{1}}^{\alpha}, c_{j_{2}}^{\alpha}, \ldots, c_{j_{n}}^{\alpha}\right)
$$

where $n=i_{1}+i_{2}+\cdots+i_{d}$ and $d \geq j_{1}, j_{2}, \ldots, j_{n} \geq 1$ is an arbitrary sequence of integers such that $\left|\left\{k \mid j_{k}=m\right\}\right|=i_{m}$. The goal of this section is to prove the following.

Proposition 2.3. Let $G \in \mathcal{G}$ be an arbitrary graph. Then $t(G)=t^{\alpha}(G)$. 
Let $G \in \mathcal{G}$ be a fixed circle free graph. A half edge in $G$ is an ordered pair $(v, e)$ of a vertex $v$ and an edge $e$ such that $v$ and $e$ are forming an incident pair. For each edge $e \in E(G)$ we introduce two half edges $h(e, 1)=\left(v_{1}, e\right)$ and $h(e, 2)=\left(v_{2}, e\right)$ where $v_{1}$ and $v_{2}$ are the two endpoints of $e$. In case $e$ is a loop, we think of $h(e, 1)$ and $h(e, 2)$ as different objects although the corresponding ordered pairs are the same. We denote by $H(G)=\{h(e, i) \mid e \in E(G), i \in\{1,2\}\}$ the set of half edges in $G$.

For each half edge $h(e, i)$, we introduce an isomorphic copy of the color space $V$ which we denote by $V_{e, i}$. In each such space $V_{e, i}$ there is a natural basis whose elements correspond to the colors $c_{1}, c_{2}, \ldots, c_{d}$. We denote the elements of this basis by $c_{1, e, i}, c_{2, e, i}, \ldots, c_{d, e, i}$. Let

$$
W=\bigotimes_{e \in E(G), i \in\{1,2\}} V_{e, i}
$$

be the tensor product of all these spaces. For the edge coloring model $t: \mathbb{N}^{d} \rightarrow R$ we define a linear form $m: W \rightarrow R$ by

$$
m\left(\bigotimes_{e \in E(G), i \in\{1,2\}} u_{e, i}\right)=\prod_{v \in V(G)} l_{d(v)}(u(v, 1), u(v, 2), \ldots, u(v, d(v)))
$$

where $u_{e, i} \in V_{e, i}$ are arbitrary elements, $d(v)$ is the degree of the vertex $v$ and $u(v, 1), u(v, 2), \ldots, u(v, d(v))$ is the list of those $u_{e, i}$-s for which the half edge $h(e, i)$ is incident to $v$. Since every half edge is incident to exactly one vertex we have that the right hand side is multi-linear in the vectors $u_{e, i}$ and thus by the universal property of the tensor product there is a unique $m$ which satisfies the equation.

Let us consider the spaces $W_{e}=V_{e, 1} \otimes V_{e, 2}$ associated to the edges of $G$. A basis of $W_{e}$ is formed by the elements $c_{i, e, 1} \otimes c_{j, e, 2}$ where $1 \leq i, j \leq d$. Thus the elements of $W_{e}$ can be represented as matrices whose rows and columns are indexed by the elements of $\mathcal{C}$. Let $J_{e}=\sum_{i=1}^{d} c_{i, e, 1} \otimes c_{i, e, 2}$ be the element of $W_{e}$ which correspond to the identity matrix and let

$$
J=\bigotimes_{e \in E(G)} J_{e} \in \bigotimes_{e \in E(G)} W_{e}=W .
$$

We have that

$$
J=\sum_{\psi: E(G) \rightarrow\{1,2, \ldots, d\}} \bigotimes_{e \in E(G), i \in\{1,2\}} c_{\psi(e), e, i} .
$$

Since the terms of this sum correspond to the colorings of the edges in $G$ it follows that

$$
t(G)=m(J) .
$$

Let $J_{0}$ denote the element $\sum_{i=1}^{d} c_{i} \otimes c_{i}$ in $V \otimes V$.

Lemma 2.4. If $b_{1}, b_{2}, \ldots, b_{d}$ is an orthonormal basis in $V$, then $J_{0}=\sum_{i=1}^{d} b_{i} \otimes b_{i}$.

Proof. Assume that $b_{j}=\sum_{i=1}^{d} a_{j, i} c_{i}$ for some real numbers $a_{j, i}$. Then the matrix $A=\left\{a_{i, j}\right\}$ is orthogonal and thus $A A^{T}=1$. Now

$$
\sum_{j=1}^{d} b_{j} \otimes b_{j}=\sum_{j=1}^{d} \sum_{i=1}^{d} \sum_{k=1}^{d} a(j, i) a(j, k) c_{i} \otimes c_{k}=\sum_{i=1}^{d} \sum_{k=1}^{d} \delta_{i, k} c_{i} \otimes c_{k}=J_{0} .
$$


Recall that $\alpha$ was an orthogonal transformation of $V$ and thus $c_{1}^{\alpha}, c_{2}^{\alpha}, \ldots, c_{d}^{\alpha}$ is an orthonormal basis in $V$. By lemma 2.4 we obtain that $\sum_{i=1}^{d} c_{i, e, 1}^{\alpha} \otimes c_{i, e, 2}^{\alpha}=J_{e}$ and so

$$
J=\sum_{\psi: E(G) \rightarrow\{1,2, \ldots, d\}} \bigotimes_{e \in E(G), i \in\{1,2\}} c_{\psi(e), e, i}^{\alpha}=J^{\alpha}
$$

We obtain that

$$
t(G)=m(J)=m\left(J^{\alpha}\right)=t^{\alpha}(G)
$$

for all circle free graphs $G$.

Now let $H \in \mathcal{G}$ be an arbitrary graph which is the disjoint union of a circle free graph $G$ and $n$ circles. The equation

$$
t(H)=t(G) d^{n}=t^{\alpha}(G) d^{n}=t^{\alpha}(H)
$$

completes the proof of proposition 2.3 .

2.6. Action of the orthogonal group on the polynomial ring $P_{d}$. Recall that $P_{d}$ is the polynomial ring $\mathbb{R}\left[\left\{x_{v} \mid v \in \mathbb{N}^{d}\right\}\right]$ and the universal edge coloring model $t_{d}$ is given by the map $t_{d}: v \rightarrow x_{v}$. For a fixed orthogonal transformation $\alpha$ of the color space $V$ we have a new edge coloring model $t_{d}^{\alpha}: \mathbb{N}^{d} \rightarrow P_{d}$. Using that $P_{d}$ is a free commutative $\mathbb{R}$-algebra with free generators $\left\{x_{v} \mid v \in \mathbb{N}^{d}\right\}$ we get that the map

$$
x_{v} \rightarrow t_{d}^{\alpha}(v)
$$

extends to an algebra endomorphism $\alpha: R_{d} \rightarrow R_{d}$. Since $\alpha^{-1}$ induces another endomorphism which is both right and left inverse for $\alpha$ it turns out that $\alpha$ is an automorphism of $R_{d}$. Proposition 2.3 implies that

Corollary 2.5. The elements of the subring $I_{d}<R_{d}$ are invariant under the action of $\alpha$ for all orthogonal transformations $\alpha$.

We define the height $h\left(x_{v}\right)$ of a variable $x_{v} \in P_{d}$ to be the sum of the components of $v$. The height of a monomial $x_{v_{1}} x_{v_{2}} \ldots x_{v_{r}}$ is defined to be the multiset $\left\{h\left(x_{v_{1}}\right), h\left(x_{v_{2}}\right), \ldots, h\left(x_{v_{r}}\right)\right\}$. We denote by $W_{S}$ the linear subspace generated by all the monomials in $P_{d}$ of height $S$. It is clear that $P_{d}$ as a vectorspace is the direct sum of the spaces $W_{S}$ where $S$ runs over all possible finite multisets of the nonnegative integers. We show that these subspaces are invariant under the action of the orthogonal group. To see this let us fix an element $\alpha \in O(V)$. Since $\left(x_{v_{1}} x_{v_{2}} \ldots x_{v_{r}}\right)^{\alpha}=x_{v_{1}}^{\alpha} x_{v_{2}}^{\alpha} \ldots x_{v_{r}}^{\alpha}$ it is enough to prove that $x_{v}^{\alpha}$ is a linear combination of variables of height $n=h\left(x_{v}\right)$ for all $v$. Let us represent $v$ by a multiset of colors $\left\{c_{i_{1}}, c_{i_{2}}, \ldots, c_{i_{n}}\right\}$. We have that

$$
x_{v}^{\alpha}=t_{d}^{\alpha}(v)=l_{n}\left(c_{i_{1}}^{\alpha}, c_{i_{2}}^{\alpha}, \ldots, c_{i_{n}}^{\alpha}\right) .
$$

By the multilinearity of $l_{n}$ the right hand side of the above equation can be written as a linear combination of some monomials of the form $l_{n}\left(c_{k_{1}}, c_{k_{2}}, \ldots, c_{k_{n}}\right)$ which are all variables of height $n$. 


\section{EXAMPLES AND QUESTIONS}

3.1. Vertex coloring models as edge coloring models. A vertex coloring model (see [4]) is given by a finite weighted graph $H$ with real edge weights $\beta_{H}(i, j)$ and positive vertex weights $\alpha_{H}(i)$. If $G$ is a simple graph, then the homomorphism function (or partition function) $\operatorname{hom}(G, H)$ is defined by

$$
\operatorname{hom}(G, H)=\sum_{\phi: V(G) \rightarrow V(H)} \prod_{v \in V(G)} \alpha_{H}(\phi(v)) \prod_{u v \in E(G)} \beta_{H}(\phi(u), \phi(v)) .
$$

We show that the graph parameter $\operatorname{hom}(G, H)$ can be represented by the partition function of an edge coloring model where the number of colors is the rank of the matrix of the edge weights in $H$. Let $B$ be the symmetric matrix of the edge weights $\beta_{H}(i, j)$. From elementary linear algebra we know that

$$
B=\lambda_{1} u_{1} u_{1}^{T}+\lambda_{2} u_{2} u_{2}^{T}+\cdots+\lambda_{r} u_{r} u_{r}^{T}
$$

for some real column vectors $u_{i}$ and numbers $\lambda_{i} \in\{1,-1\}$ where $r$ is the rank of $B$. Let $t$ be the edge coloring model given by

$$
t\left(s_{1}, s_{2}, \ldots, s_{r}\right)=\sum_{j=1}^{|V(H)|} \alpha_{H}(j) \prod_{i=1}^{r}\left(u_{i}(j) \sqrt{\lambda_{i}}\right)^{s_{i}} .
$$

Using the fact that $\beta_{H}(i, j)=\sum_{k=1}^{r} u_{k}(i) u_{k}(j) \lambda_{k}$ we get that $t(G)=\operatorname{hom}(G, H)$ for an arbitrary simple graph $G$. It is worth mentioning that if $B$ is positive semidefinite, then the numbers $\lambda_{i}$ are all 1 and the representing edge coloring model is real valued. However there are cases when the edge coloring model is real valued without this condition. A simple example is the Ising model which can be represented by a weighted graph on 2 vertices with $\beta(1,1)=\beta(2,2)=a \geq 0$, $\beta(1,2)=\beta(2,1)=b \geq 0$ and $\alpha(1)=\alpha(2)=1$. It is easy to compute that the corresponding edge coloring model is given by

$$
\begin{aligned}
t\left(s_{1}, s_{2}\right)= & 2\left(\frac{a+b}{2}\right)^{\frac{s_{1}}{2}}\left(\frac{a-b}{2}\right)^{\frac{s_{2}}{2}} \text { if } s_{2} \text { is even } \\
& t\left(s_{1}, s_{2}\right)=0 \text { if } s_{2} \text { is odd. }
\end{aligned}
$$

It is an interesting phenomenon that the number of colors needed to represent a vertex coloring model by an edge coloring model depends only on the rank of the adjacency matrix of the weighted graph. This leads to a family of infinite vertex coloring models which are still representable by ordinary edge coloring models. Let $w:[0, a]^{2} \rightarrow \mathbb{R}$ be a bounded symmetric measurable function such that

$$
w(x, y)=\sum_{i=1}^{r} \lambda_{i} f_{i}(x) f_{i}(y)
$$

for some bounded measurable functions $f_{i}$ and numbers $\lambda_{i} \in\{1,-1\}$. Regarding the function $w$ as an infinite adjacency matrix one can define an analogy of the homomorphism function by

$$
t_{w}(G)=\int_{x_{1}, x_{2}, \ldots, x_{m}} \prod_{(i, j) \in E(G), i<j} w\left(x_{i}, x_{j}\right) d x_{1} d x_{2} \ldots d x_{m}
$$

where $G$ is an arbitrary graph with $|V(G)|=m$ such that the vertices of $G$ are indexed by the numbers $\{1,2, \ldots, m\}$. Note that $t_{w}$ is a vertex reflection positive 
and multiplicative graph parameter. Let us introduce the following edge coloring model:

$$
t\left(s_{1}, s_{2}, \ldots, s_{r}\right)=\int_{x \in[0, a]} \prod_{i=1}^{r}\left(f_{i}(x) \sqrt{\lambda_{i}}\right)^{s_{i}} d x .
$$

It can be easily calculated that $t_{w}(G)=t(G)$ and that if $\lambda_{i}=1$ for all $i$, then $t$ gives rise to a real valued edge coloring model.

3.2. Graph parameters from combinatorics. Many interesting graph parameters can be obtained from the following special family of edge coloring models. Let $S$ be a subset of $\mathbb{N}^{r}$ and let $t_{S}: \mathbb{N}^{r} \rightarrow \mathbb{R}$ be the function with $t_{S}(v)=1$ if $s \in S$ and $t_{S}(v)=0$ if $s \notin S$. Let $G$ be a simple graph. The next table lists a few examples.

\begin{tabular}{c|c}
$\mathrm{S}$ & combinatorial meaning of $t_{S}(G)$ \\
$\{1\} \times \mathbb{N}$ & number of perfect machings \\
$\{2\} \times \mathbb{N}$ & number of fully packed loop configurations \\
$\{0,1\} \times \mathbb{N}$ & number of matchins \\
$\{0,2\} \times \mathbb{N}$ & number of loop configurations \\
$\{0, d\} \times \mathbb{N}$ & number of $d$-regular subgraphs \\
$\{0,1\}^{d}$ & number of proper edge colorings with $d$ colors \\
$\{(b, c, d) \mid b+d \equiv c+d \equiv 0(2)\}$ & number of nowhere zero 4-flows \\
$\{(2,0,0),(0,2,0),(0,0,1)\} \times \mathbb{N}$ & permanent of the adjacency matrix
\end{tabular}

Using the orthogonal invariance of partition functions one can create peculiar equations. For example by rotating the first example on the list with 45-degree we get the edge coloring model

$$
t(a, b)=\sqrt{2}^{-(a+b)}(a-b)
$$

whose partition function is again the number of perfect matchings. In other words, the partition function of the model $t(a, b)=a-b$ is $2^{|E(G)|}$ times the number of perfect matchings in $G$.

3.3. Open questions. Let $f$ be an edge coloring model with $d$ colors and let $M(k, f)$ denote its $k$-th connection matrix (see section 2.2). It is not hard to see 5 that $\operatorname{rk}(M(k, f)) \leq d^{k}$.

Question 3.1. What are the possible sequences $\operatorname{rk}(M(k, f)), k=1,2,3, \ldots$ ?

The analogy of this question for vertex coloring models was answered by L. Lovász in [6].

The next question is motivated by section 3.1 .

Question 3.2. Which are those vertex coloring models whose partition functions are edge reflection positive?

We know only two examples: The Ising model and the vertex coloring models with positive semidefinite adjacency matrices.

\section{Proof of the characterization theorem}

Throughout this section we prove theorem 2.2. We assume that $f$ is an edgereflection positive and multiplicative graph parameter. Recall that our goal is to construct an edge coloring model $t: \mathbb{N}^{d} \rightarrow \mathbb{R}$ for some integer $d$ such that the corresponding partition function equals $f$. 
4.1. The value of a circle. Let $k$ be a natural number and let $\mathcal{M}_{k}$ denote the set of those graphs $G$ in $\mathcal{G}_{k}$ which are circle free and $V(G)=\emptyset$. In particular the edge set of $G$ is a perfect matching on the $k$ open ends. It follows that if $k$ is an odd number, then $\mathcal{M}_{k}$ is empty. We denote by $\mathcal{Q M}_{k}$ the subspace generated by $\mathcal{M}_{k}$ in $\mathcal{Q}_{k}$.

Assume that $k=2 n$ for some natural number $n$ and let $A_{k}$ denote the subset of all elements $G$ of $\mathcal{M}_{k}$ with the property that each edge of $G$ connects an open end with label $\leq n$ and another open end with label $>n$. The elements of $A_{k}$ can be represented by permutations of the set $\{1,2, \ldots, n\}$ in a way that a permutation $\pi$ corresponds to a matching $a_{\pi} \in A_{k}$ where the open end $i$ is connected with $\pi(i)+n$ for all $1 \leq i \leq n$. Now the definition of gluing implies that $g\left(a_{\pi}, a_{\varrho}\right)$ is a graph which is the disjoint union of $c\left(\pi \varrho^{-1}\right)$ circles where $c(\sigma)$ denotes the number of cycles in a permutation $\sigma$. Let $d$ be the value of $f$ on a single circle. Using the multiplicativity of $f$ we have that

$$
f\left(g\left(a_{\pi}, a_{\varrho}\right)\right)=d^{c\left(\pi \varrho^{-1}\right)} .
$$

Let $M_{n}$ be a matrix whose rows and columns are indexed by permutations from the symmetric group $S_{n}$ and from the entry in the intersection if the row corresponding to $\pi$ and column corresponding to $\varrho$ is $d^{c\left(\pi \varrho^{-1}\right)}$. The assumption that $f$ is reflection positive implies that $M_{n}$ must be a positive semidefinite matrix for every $n$. We will prove that this is only possible if $d$ is a nonnegative integer.

The matrix $M_{n}$ is acting on the space of formal linear combinations of the group elements of $S_{n}$ which is the group algebra $\mathbb{R}\left[S_{n}\right]$. Let

$$
w=\sum_{\pi \in S_{n}} \operatorname{sgn}(\pi) \pi
$$

We have that

$$
\begin{aligned}
w M_{k} & =\sum_{\pi, \varrho \in S_{n}} \operatorname{sgn}(\pi) d^{c\left(\pi \varrho^{-1}\right)} \varrho=\sum_{\pi, \varrho \in S_{n}} \operatorname{sgn}\left(\pi \varrho^{-1}\right) d^{c\left(\pi \varrho^{-1}\right)} \operatorname{sgn}(\varrho) \varrho \\
& =w\left(\sum_{\pi \in S_{n}} \operatorname{sgn}(\pi) d^{c(\pi)}\right) .
\end{aligned}
$$

This means that $w$ is an eigenvector of $M_{n}$ with eigenvalue

$$
\sum_{\pi \in S_{n}} \operatorname{sgn}(\pi) d^{c(\pi)}=d(d-1)(d-2) \ldots(d-n+1) .
$$

The positive semidefinitness of $M_{n}$ implies that $d(d-1) \ldots(d-n+1)$ must be a nonnegative number for every natural number $n$ and so $d$ is a nonnegative integer.

As the next lemma shows, the fact that the circle value is a nonnegative integer is the first step towards the existence of an edge coloring model representing $f$.

Lemma 4.1. Let $t$ be an arbitrary edge coloring model with $d$ colors. Then

$$
f(g(H, K))=t(g(H, K))
$$

for every pair $H, K \in \mathcal{Q M}_{k}$.

Proof. The quantum graph $g(H, K)$ is the linear combination of graphs consisting only of circles. The multiplicativity of $f$ shows that the value of $t$ and $f$ must be the same on such a graph. 
Using the terminology of section 2.3. we have that

Lemma 4.2. Let $t$ be any edge coloring model with $d$ colors and let $H \in \mathcal{Q M}_{k}$. Then $f(g(H, H))=t(g(H, H))=0$ if and only if $t_{\chi}(H)=0$ for all colorings $\chi:\{1,2, \ldots, k\} \rightarrow \mathcal{C}$.

4.2. Lifting to the universal edge coloring model. Recall that $d$ is the circle value of $f$ and $t_{d}$ is the universal edge coloring model with $d$ colors. In this section we prove that

Lemma 4.3. If $Q \in \mathcal{Q}$ is an arbitrary quantum graph and $t_{d}(Q)=0$, then $f(Q)=$ 0 .

Proof. For a graph $G \in \mathcal{G}$ we define its height $h(G)$ to be the multiset of the degrees of the vertices in $G$. From the definition of the universal edge coloring model it follows that $t_{d}(G) \in W_{h(G)}$. Every quantum graph $Q \in \mathcal{Q}$ can be written in the form $\sum_{S} Q_{S}$ where $Q_{S}$ is a quantum graph which is a linear combination of graphs of height $S$. We have that $t_{d}(Q)=\sum_{S} t_{d}\left(Q_{S}\right)$ and $t_{d}\left(Q_{S}\right) \in W_{S}$ for every multiset $S$. It follow that $t_{d}(Q)=0$ implies that $t_{d}\left(Q_{S}\right)=0$ for all multisets $S$. Thus we can assume that $Q$ is homogeneous in the sense that each graph component of $Q$ has the same height $S$.

Let $G \in \mathcal{G}$ be a graph which is the disjoint union of a circle free graph $H$ and $n$ circles. Both $f$ and $t_{d}$ vanish on the quantum graph $G-d^{n} H$. This means that one can eliminate circles in a quantum graph without changing the value of $f$ and $t_{d}$ on it. Thus we can assume that $Q$ is a combination of circle free graphs.

Assume that $S$ consists of $n$ numbers and their sum is $k$. Let $G_{S}$ be a graph in $\mathcal{G}_{k}$ with the following properties:

1. $\left|V\left(G_{S}\right)\right|=n$.

2. $\left|E\left(G_{S}\right)\right|=|O(G)|=k$.

3. Each edge $e \in E(G)$ connects an open end with a vertex.

4. The multiset of the degrees of the verteces is $S$.

It is clear that $G_{S}$ is unique up to a relabeling of the open ends. It is also clear that for every graph $G$ of height $S$ there is a matching $M \in \mathcal{M}_{k}$ such that $G=g\left(G_{S}, M\right)$. This implies that our quantum graph $Q$ can be written in the form $g\left(G_{S}, M\right)$ where $M$ is in $\mathcal{Q} \mathcal{M}_{k}$.

Let $\mathcal{P}_{v} \subseteq O(G)$ denote the set of those open ends which are connected to the vertex $v \in V(G)$ in $G_{S}$ and let $\mathcal{P}=\left\{\mathcal{P}_{v} \mid v \in V(G)\right\}$ be the partition formed by these sets. We denote by $K \leq S_{k}$ the automorphism group of $\mathcal{P}$. It is clear that $g\left(G_{S}, M\right)$ is isomorphic to $g\left(G_{S}, M^{\sigma}\right)$ for all $\sigma \in K$. Let

$$
\hat{M}=\frac{1}{|K|} \sum_{\sigma \in K} M^{\sigma}
$$

We have that

$$
f(Q)=f\left(g\left(G_{S}, M\right)\right)=f\left(g\left(G_{S}, \hat{M}\right)\right)
$$

and

$$
0=t_{d}(Q)=t_{d}\left(g\left(G_{S}, M\right)\right)=t_{d}\left(g\left(G_{S}, \hat{M}\right)\right) .
$$

Using equation (11) from section 2.3 we get that

$$
0=t_{d}(Q)=\sum_{\chi:\{1,2, \ldots, k\} \rightarrow \mathcal{C}} t_{d \chi}\left(G_{S}\right) t_{d \chi}(\hat{M})
$$


The group $K$ is acting on the colorings $\chi:\{1,2, \ldots, k\} \rightarrow \mathcal{C}$. An orbit of this action can be described as a multiset $X_{\chi}$ of multisets such that the elements of $X_{\chi}$ are multisets of colors describing the color distributions in different partition sets of $\mathcal{P}$. The value of $t_{d_{\chi}}\left(G_{S}\right)$ depends only on the orbit of $\chi$ because $G_{S}$ and $G_{S}^{\sigma}$ are isomorphic for every $\sigma \in K$. Moreover $t_{d \chi}\left(G_{S}\right)$ is a monomial of height $S$ of the form $x_{v_{1}} x_{v_{2}} \ldots x_{v_{n}}$ where the vectors $v_{i}$ describe multisets of colors which can be seen at different vertices and the list $v_{1}, v_{2}, \ldots, x_{n}$ describes $X_{\chi}$. On the other hand $t_{d \chi}(\hat{M})$ is a real number which also depends only the orbit of $\chi$ because $\hat{M}$ is $K$ symmetric. By using the fact that different monomials are linearly independent over $\mathbb{R}$ we obtain that $t_{d \chi}(\hat{M})$ must be 0 for all colorings $\chi$. This implies by lemma 4.2 that $t_{d}(g(\hat{M}, \hat{M}))=0$ and so by lemma 4.1 we get that $f(g(\hat{M}, \hat{M}))=0$.

Since $f(g(-,-))$ is a positive semidefinite form it follows that $f(g(Y, \hat{M}))=0$ for all $Y \in \mathcal{G}_{k}$. In particular

$$
f(Q)=f\left(g\left(G_{S}, \hat{M}\right)\right)=0 .
$$

Corollary 4.4. There exists a homomorphism $\hat{f}: I_{d} \rightarrow \mathbb{R}$ such that $f(Q)=$ $\hat{f}\left(t_{d}(Q)\right)$ for every quantum graph $Q \in \mathcal{Q}$.

Proof. Recall that $\mathcal{Q}$ has an $\mathbb{R}$-algebra structure and that $I_{d}$ is the image of $\mathcal{Q}$ under the algebra homomorphism $t_{d}: \mathcal{Q} \rightarrow P_{d}$. On the other hand $f: \mathcal{Q} \rightarrow \mathbb{R}$ is an algebra homomorphism because $f$ is multiplicative. According to the main result of this section we have that

$$
\operatorname{ker}\left(t_{d}\right) \subseteq \operatorname{ker}(f) \subseteq \mathcal{Q},
$$

and this completes the proof.

4.3. Representing invariants of the orthogonal group with quantum graphs. The main result of this section is the following.

Lemma 4.5. If $p \in P_{d}$ is invariant under the action of the orthogonal group $O_{d}(\mathbb{R})$, then there is a quantum graph $Q \in \mathcal{Q}$ such that $t_{d}(Q)=p$.

Before we start proving lemma 4.5 we describe a construction which will be useful in this and in later sections.

Tensor construction. Let $X$ be an arbitrary finite set with a partition $\mathcal{Y}=$ $\left\{Y_{1}, Y_{2}, \ldots, Y_{n}\right\}$ on its elements. Let $V_{x}$ be an isomorphic copy of the color-space $V=\left\langle c_{1}, c_{2}, \ldots, c_{d}\right\rangle_{\mathbb{R}}$ for each element $x \in X$ and let

$$
T(X, \mathcal{Y})=\bigotimes_{x \in X} V_{x}
$$

Let $l_{n}$ denote the symmetric $n$-linear form from section 2.5 associated with the universal edge coloring model $t_{d}$. For each partition set $Y_{i}$ we define a multilinear form $\hat{m}_{i}$ by applying $l_{\left|Y_{i}\right|}$ for the spaces $\left\{V_{x} \mid x \in Y_{i}\right\}$. The product $\prod_{i=1}^{k} \hat{m}_{i}$ defines a multilinear form in the spaces $\left\{V_{x} \mid x \in X\right\}$. By factoring $\hat{m}$ trough the tensor product $T(X, \mathcal{Y})$ we get an $\mathbb{R}$-linear form $m: T(X, \mathcal{Y}) \rightarrow P_{d}$. The space $T(X, \mathcal{Y})$ admits a euclidean scalar product which comes from the euclidean structure on $V$. An orthonormal basis for this scalar product is formed by the different tensor products of the color vectors. This basis will be called the color basis. The orthogonal group $O_{d}(\mathbb{R})$ which preserves the scalar product on $V$ is also acting on $T(X, \mathcal{Y})$ by taking the tensor product of the actions on $V_{x}$. This action has the property that 
$m\left(t^{\alpha}\right)=m(t)^{\alpha}$ where $t \in T(X, \mathcal{Y})$ and $\alpha \in O_{d}(\mathbb{R})$. Let $S=\left\{\left|Y_{1}\right|,\left|Y_{2}\right|, \ldots,\left|Y_{n}\right|\right\}$ be the multiset of the sizes of the partition sets. If we substitute color vectors into the multilinear form $\hat{m}$ we get all the monomials of height $S$ in $P_{d}$. It follows that $m$ maps $T(X, \mathcal{Y})$ to $W_{s}$ surjectively.

Proof of Lemma 4.5. We know from section 2.6 that $P_{d}$ is the direct sum (as a vectorspace) of the spaces $W_{S}$ where each $W_{S}$ is invariant (as a subspace) under the action of $O_{d}(\mathbb{R})$. It follows that if $p$ is an invariant element of $O_{d}(\mathbb{R})$, then each $W_{S}$ component $p_{S}$ of $p$ must be invariant too. Since $p$ is the sum of its $W_{S}$ components it is enough to find quantum graphs $Q_{S}$ with $t_{d}\left(Q_{S}\right)=p_{S}$ for each multiset $S$.

Let $S=\left\{s_{1}, s_{2}, \ldots, s_{n}\right\}$ be a fixed multiset of natural numbers and let $k=\sum_{i} s_{i}$. Let $\mathcal{D}=\left\{D_{1}, D_{2}, \ldots, D_{n}\right\}$ be a partition of the index set $\{1,2, \ldots, k\}$ such that $\left|D_{i}\right|=s_{i}$ for all $1 \leq i \leq n$. Let $W=T(\{1,2, \ldots, k\}, \mathcal{D})$ and let $W^{0}$ be the kernel of the map $m$. We have that the space $W^{0}$ is invariant under $O_{d}(\mathbb{R})$ and $W / W^{0}$ is isomorphic to $W_{S}$ in a way that the induced action of $O_{d}(\mathbb{R})$ on $W / W^{0}$ commutes with this isomorphism. By abusing the notation we identify $W_{S}$ with $W / W^{0}$.

Let $p_{1}$ be a preimage of $p_{S}$ under the homomorphism $W \rightarrow W / W^{0}$ and let

$$
\bar{p}=\int_{\alpha \in O_{d}(\mathbb{R})} p_{1}^{\alpha} d \nu
$$

where $\nu$ is the normalized Haar measure on the orthogonal group $O_{d}(\mathbb{R})$. Since $p_{S}$ is invariant in $W_{S}=W / W^{0}$ it follows that $\bar{p}$ is also a preimage of $p_{S}$ under the map $W \rightarrow W / W^{0}$. Furthermore we have that $\bar{p}$ is an invariant of $O_{d}(\mathbb{R})$.

The first fundamental theorem of Weyl [8] describes the space of invariant elements in $W$ by determining a generating system for it. The elements of this generating system correspond to partitions of the set $\{1,2, \ldots, k\}$ into two element subsets. In particular if $k$ is an odd number, then the only invariant is the zero vector. Assume that $k$ is even and let $\mathcal{E}=\left\{E_{1}, E_{2}, \ldots, E_{k / 2}\right\}$ be such a partition. Let $\chi: \mathcal{E} \rightarrow \mathcal{C}$ be a coloring of the partition sets. The function $\chi$ induces a coloring $\hat{\chi}:\{1,2, \ldots, k\} \rightarrow \mathcal{C}$ such that $\hat{\chi}(j)=\chi\left(E_{i_{j}}\right)$ for $1 \leq j \leq k$ where $i_{j}$ denotes the number for which $j \in E_{i_{j}}$. We define $g_{\chi}$ to be the tensor product

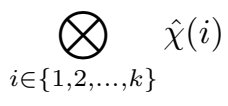

where $\hat{\chi}(i) \in V_{i}$. The invariant which correspond to $\mathcal{E}$ is

$$
g=\sum_{\chi: \mathcal{E} \rightarrow \mathcal{C}} g_{\chi}
$$

We define a graph $G \in \mathcal{G}$ associated to the invariant $g$. Let $V(G)=\left\{v_{1}, v_{2}, \ldots, v_{n}\right\}$ and $E(G)=\left\{e_{1}, e_{2}, \ldots, e_{k / 2}\right\}$. The edge $e_{i}$ connects the vertices $v_{i_{1}}$ and $v_{i_{2}}$ where one element of $E_{i}$ is in the partition set $D_{i_{1}}$ and the other element of $E_{i}$ is in the partition set $D_{i_{2}}$. In other words, the vertices of $G$ correspond to the partition sets in $\mathcal{D}$, the edges correspond to the partition sets in $\mathcal{E}$ and the edge corresponding to $E_{i}$ is incident to the vertex corresponding to $D_{j}$ if and only if $E_{1} \cap D_{j} \neq \emptyset$.

Now the spaces $V_{i}$ are in a one to one correspondence with the half edges in $G$ and the form $m$ coincides with the one defined in section 2.5. It follows that $m(g)=t_{d}(G)$. 
Using Weyl's theorem we have that $\bar{p}=\sum_{i=1}^{r} \lambda_{i} g_{i}$ for some real numbers $\lambda_{i}$ and invariants $g_{i}$ where for each $g_{i}$ there is a graph $G_{i} \in \mathcal{G}$ with $m\left(g_{1}\right)=t_{d}\left(G_{i}\right)$. It follows that

$$
p_{S}=m(\bar{p})=\sum_{i=1}^{r} \lambda_{i} t_{d}\left(G_{i}\right)=t_{d}\left(\sum_{i=1}^{r} \lambda_{i} G_{i}\right) .
$$

4.4. Projection to subalgebras of the matrix algebra. Let $A$ be a subalgebra of the full matrix algebra $\mathbb{M}_{n}(\mathbb{R})$ such that $A=\left\{M^{T} \mid M \in A\right\}$. The bilinear function $(M, K)=\operatorname{tr}\left(M K^{T}\right)$ defines a euclidean scalar product on $\mathbb{M}_{n}(\mathbb{R})$. Let $\mathcal{P}_{A}$ denote the orthogonal projection to $A$.

Lemma 4.6. If $M$ is a symmetric positive semidefinite matrix, then $\mathcal{P}_{A}(M)=K^{2}$ for some symmetric matrix $K \in A$.

Proof. Since $A$ is invariant under transposing, we have that $\mathcal{P}_{A}(M)$ is a symmetric matrix in $A$. First we prove that the eigenvalues of $\mathcal{P}_{A}(M)$ are all nonnegative. Let $\lambda_{1}, \lambda_{2}, \ldots, \lambda_{k}$ be the set of the positive eigenvalues of $\mathcal{P}_{A}(M)$, let $p(x)=(x-$ $\left.\lambda_{1}\right)\left(x-\lambda_{2}\right) \ldots\left(x-\lambda_{k}\right)$ and let $H=p\left(\mathcal{P}_{A}(M)\right)$. Using the fact that $\mathcal{P}_{A}$ is self adjoint and that $H^{T}=H \in A$, we have that

$$
\begin{aligned}
\operatorname{tr}\left(\mathcal{P}_{A}(M) H^{2}\right) & =\left(\mathcal{P}_{A}(M), H^{2}\right)=\left(M, \mathcal{P}_{A}\left(H^{2}\right)\right) \\
& =\left(M, H^{2}\right)=\operatorname{tr}\left(M H^{2}\right)=\operatorname{tr}(H M H) \geq 0 .
\end{aligned}
$$

Since $\operatorname{tr}\left(\mathcal{P}_{A}(M) H^{2}\right)$ is a positive linear combination of the negative eigenvalues of $\mathcal{P}_{A}(M)$ it follows that the eigenvalues of $\mathcal{P}_{A}(M)$ must be all nonnegative.

Let $g \in \mathbb{R}[x]$ be a polynomial such that $g\left(\lambda_{i}\right)=\sqrt{\lambda_{i}}$ for $1 \leq i \leq k$. Now $K=g\left(\mathcal{P}_{A}(M)\right)$ satisfies both $K^{2}=\mathcal{P}_{A}(M)$ and $K \in A$.

4.5. Genaralized Brauer algebras. Let $\mathcal{S}$ denote the set of finite multisets of the positive integers. Let $\mu(S)$ denote the sum of the elements of a multiset $S \in \mathcal{S}$. For each multiset $S \in \mathcal{S}$ we introduce a set $O(S)$ of size $\mu(S)$ and we define a partition $P(S)$ on the elements of $O(S)$ such that the multiset of the sizes of the partition sets in $P(S)$ is $S$. The algebra $A_{d}$ consists of the formal linear combinations of triples

$$
a\left(S_{1}, S_{2}, M\right)
$$

where $S_{1}, S_{2} \in \mathcal{S}$ and $M$ is a perfect matching on the set $O\left(S_{1}\right) \cup O\left(S_{2}\right)$. The product

$$
a\left(S_{1}, S_{2}, M_{1}\right) a\left(S_{3}, S_{4}, M_{2}\right)
$$

is defined to be 0 if $S_{2} \neq S_{3}$. If $S_{2}=S_{3}$, then $M_{1} \cup M_{2}$ is the edge set of a graph $G$ with node set $O\left(S_{1}\right) \cup O\left(S_{2}\right) \cup O\left(S_{4}\right)$ such that nodes in $O\left(S_{2}\right)$ have degree 2 and nodes in $O\left(S_{1}\right) \cup O\left(S_{4}\right)$ have degree 1 . This means that $G$ is the union of node disjoint paths and cycles. Replacing each path by a single edge we get a matching $M_{3}$ on $O\left(S_{1}\right) \cup O\left(S_{4}\right)$. Assume that the number of cycles in $G$ is $n$. The product $a\left(S_{1}, S_{2}, M_{1}\right) a\left(S_{2}, S_{4}, M_{2}\right)$ is defined to be $d^{n} a\left(S_{1}, S_{4}, M_{3}\right)$.

We introduce the transpose map on $A_{d}$ as the unique linear extension of the map

$$
a\left(S_{1}, S_{2}, M\right)^{T}=a\left(S_{2}, S_{1}, M\right) .
$$

Let $A_{d}\left(S_{1}, S_{2}\right)$ denote the space spanned by the elements $a\left(S_{1}, S_{2}, M\right)$ where $M$ runs through all perfect matchings of $O\left(S_{1}\right) \cup O\left(S_{2}\right)$. We have that

$$
A_{d}=\bigoplus_{S_{1}, S_{2} \in \mathcal{S}} A_{d}\left(S_{1}, S_{2}\right) \text {. }
$$


Let

$$
A_{d}(S)=\bigoplus_{S_{1} \in \mathcal{S}} A_{d}\left(S_{1}, S\right)
$$

and

$$
A_{d}(S)^{T}=\bigoplus_{S_{1} \in \mathcal{S}} A_{d}\left(S, S_{1}\right) .
$$

For an arbitrary basis element $a\left(S_{1}, S_{2}, M\right)$ we define $\tau\left(a\left(S_{1}, S_{2}, M\right)\right) \in \mathcal{G}$, $\tau_{1}\left(a\left(S_{1}, S_{2}, M\right)\right) \in \mathcal{G}_{\mu\left(S_{2}\right)}$ and $\tau_{2}\left(a\left(S_{1}, S_{2}, M\right)\right) \in \mathcal{G}_{\mu\left(S_{1}\right)}$ in the following way. By identifying nodes in $O\left(S_{1}\right) \cup O\left(S_{2}\right)$ belonging to the same partition set of $P\left(S_{1}\right) \cup P\left(S_{2}\right)$ we get $\tau\left(a\left(S_{1}, S_{2}, M\right)\right)$. By identifying nodes in $O\left(S_{1}\right)\left(\operatorname{resp} O\left(S_{2}\right)\right)$ belonging to the same partition set of $P\left(S_{1}\right)$ (resp. $P\left(S_{2}\right)$ ) and defining $O\left(S_{2}\right)$ (resp. $\left.O\left(S_{1}\right)\right)$ to be the set of open edges we get $\tau_{1}\left(a\left(S_{1}, S_{2}, M\right)\right)$ (resp. $\tau_{2}\left(a\left(S_{1}, S_{2}, M\right)\right)$ ). The map $\tau$ extends linearly to a map $\tau: A_{d} \rightarrow \mathcal{Q}$ and the maps $\tau_{1}, \tau_{2}$ extend to maps

$$
\tau_{1}: A_{d}(S) \rightarrow \mathcal{Q}_{\mu(S)}, \tau_{2}: A_{d}(S)^{T} \rightarrow \mathcal{Q}_{\mu(S)} .
$$

Lemma 4.7. If $b \in A_{d}$, then $f\left(\tau\left(b b^{T}\right)\right) \geq 0$.

Proof. We use the fact that

$$
b=\sum_{S \in \mathcal{S}, \mu(S) \leq m} b_{S}
$$

where $b_{S} \in A_{d}(S)$ for all $S$ and $m$ is a large enough natural number. Since

$$
b b^{T}=\sum_{S \in \mathcal{S}, \mu(S) \leq m} b_{S} b_{S}^{T}
$$

it suffices to show that $f\left(\tau\left(b_{S} b_{S}^{T}\right)\right) \geq 0$ for all $S$. Using the fact that $\tau_{1}\left(b_{S}\right)=\tau_{2}\left(b_{S}^{T}\right)$ we obtain that $Q=g\left(\tau_{1}\left(b_{S}\right), \tau_{2}\left(b_{S}^{T}\right)\right)$ is a reflection symmetric quantum graph. The graph $\tau\left(b_{S} b_{S}^{T}\right)$ can be obtained from $Q$ by a process where in each step we delete a circle from a graph component and multiply it by $d$. Using the fact that the circle value of $f$ is $d$, and that $f$ is multiplicative and edge reflection positive, we have that

$$
f\left(\tau\left(b_{S} b_{S}^{T}\right)\right)=f(Q) \geq 0 .
$$

Now we describe a matrix representation of the algebra $A_{d}$ which will be of crucial importance in the next section. Let us introduce the notation $\mathbb{M}(X, Y)$ for the space of real matrices whose rows are indexed by the set $X$ and whose columns are indexed by the set $Y$ where $X$ and $Y$ are finite sets. Recall that $\mathcal{C}=\left\{c_{1}, c_{2}, \ldots, c_{d}\right\}$ is a set with $d$ colors. Let $S_{1}, S_{2} \in \mathcal{S}$ be two multisets and let $M$ be a perfect matching on $O\left(S_{1}\right) \cup O\left(S_{2}\right)$. We say that a coloring of $O\left(S_{1}\right) \cup O\left(S_{2}\right)$ is compatible with $M$ if the two endpoints of each matching edge have the same color. First we represent $a\left(S_{1}, S_{2}, M\right)$ by a matrix whose rows are indexed by colorings $O\left(S_{1}\right) \rightarrow \mathcal{C}$ and whose columns are indexed by colorings $O\left(S_{2}\right) \rightarrow \mathcal{C}$. The entry in the intersection of the row $\chi$ and column $\psi$ is 1 if the coloring $\chi \times \psi: O\left(S_{1}\right) \cup O\left(S_{2}\right) \rightarrow \mathcal{C}$ is compatible with $M$ and is 0 otherwise. By extending this representation linearly to $A_{d}\left(S_{1}, S_{2}\right)$ we obtain a map

$$
\omega: A_{d}\left(S_{1}, S_{2}\right) \rightarrow \mathbb{M}\left(\mathcal{C}^{O\left(S_{1}\right)}, \mathcal{C}^{O\left(S_{2}\right)}\right) .
$$

The reader can easily check that the map $\omega$ satisfies the identity

$$
\omega\left(a\left(S_{1}, S_{2}, M_{1}\right) a\left(S_{2}, S_{3}, M_{2}\right)\right)=\omega\left(a\left(S_{1}, S_{2}, M_{1}\right)\right) \omega\left(a\left(S_{2}, S_{3}, M_{2}\right)\right) .
$$


It follows that $\omega\left(b_{1} b_{2}\right)=\omega\left(b_{1}\right) \omega\left(b_{2}\right)$ if $b_{1} \in A_{d}\left(S_{1}, S_{2}\right)$ and $b_{2} \in A_{d}\left(S_{2}, S_{3}\right)$. Let

$$
\hat{A}_{d}\left(S_{1}, S_{2}\right)=\mathbb{M}\left(\mathcal{C}^{O\left(S_{1}\right)}, \mathcal{C}^{O\left(S_{2}\right)}\right)
$$

and let

$$
\hat{A}_{d}=\bigoplus_{S_{1}, S_{2} \in \mathcal{S}} \hat{A}_{d}\left(S_{1}, S_{2}\right)
$$

The space $\hat{A}_{d}$ is endowed with a natural algebra structure in the following way. Assume that

$$
b_{1} \in \hat{A}_{d}\left(S_{1}, S_{2}\right), b_{2} \in \hat{A}_{d}\left(S_{3}, S_{4}\right) .
$$

If $S_{2}=S_{3}$, then $b_{1} b_{2}$ is the usual matrix product and if $S_{2} \neq S_{3}$, then $b_{1} b_{2}$ is defined to be 0 . This multiplication rule defines a multiplication on the whole space $\hat{A}_{d}$. It is clear that the map $\omega$ extends to an algebra homomorphism $\omega: A_{d} \rightarrow \hat{A}_{d}$. Let

$$
A_{d, r}=\bigoplus_{S_{1}, S_{2} \in \mathcal{S},} \bigoplus_{\mu\left(S_{1}\right), \mu\left(S_{2}\right) \leq r} A_{d}\left(S_{1}, S_{2}\right)
$$

and let

$$
\hat{A}_{d, r}=\bigoplus_{S_{1}, S_{2} \in \mathcal{S},} \bigoplus_{\mu\left(S_{1}\right), \mu\left(S_{2}\right) \leq r} \hat{A}_{d}\left(S_{1}, S_{2}\right) .
$$

The space $A_{d, r}$ is a subalgebra of $A_{d}$ and the space $\hat{A}_{d, r}$ is a subalgebra of $\hat{A}_{d}$. Moreover, $\omega$ maps $A_{d, r}$ into $\hat{A}_{d, r}$. It is easy to see that $\hat{A}_{d, m}$ is the full matrix algebra

$$
\mathbb{M}\left(\bigcup_{S \in \mathcal{S}, \mu(S) \leq r} \mathcal{C}^{O(S)}, \bigcup_{S \in \mathcal{S}, \mu(S) \leq r} \mathcal{C}^{O(S)}\right)
$$

and that $\omega\left(b^{T}\right)=\omega(b)^{T}$ for all $b \in A_{d, r}$. This implies in particular that $\omega\left(A_{d, r}\right)$ is a subalgebra of the matrix algebra $\hat{A}_{d, r}$ which is closed under taking transpose. Let us define the euclidean scalar product $\left(b_{1}, b_{2}\right)=\operatorname{tr}\left(b_{1} b_{2}\right)$ on $\hat{A}_{d, r}$. The spaces $\hat{A}_{d}\left(S_{1}, S_{2}\right)$ are orthogonal to each other in $\hat{A}_{d, r}$ for different pairs $\left(S_{1}, S_{2}\right)$. Since $\omega\left(A_{d}\left(S_{1}, S_{2}\right)\right)$ is contained in $\hat{A}_{d}\left(S_{1}, S_{2}\right)$ we have that

$$
\omega\left(A_{d, r}\right)=\bigoplus_{S_{1}, S_{2} \in \mathcal{S}, \mu\left(S_{1}\right), \mu\left(S_{2}\right) \leq r} \omega\left(A_{d}\left(S_{1}, S_{2}\right)\right)
$$

where all the direct summands are orthogonal to each other. Let $\mathcal{P}_{d, r}$ denote the orthogonal projection of $\hat{A}_{d, r}$ to $\omega\left(A_{d, r}\right)$ and let $\mathcal{P}_{d, r}\left(S_{1}, S_{2}\right)$ denote the orthogonal projection of $\hat{A}_{d, r}$ to $\omega\left(A_{d}\left(S_{1}, S_{2}\right)\right)$ where $\mu\left(S_{1}\right), \mu\left(S_{2}\right) \leq r$. The above properties imply that the restriction of $\mathcal{P}_{d, r}$ to the space $\hat{A}_{d}\left(S_{1}, S_{2}\right)$ is $\mathcal{P}_{d, r}\left(S_{1}, S_{2}\right)$.

Now we use the tensor construction from section 4.3 . Let us observe that the elements of the color basis in the space $T\left(O\left(S_{1}\right) \cup O\left(S_{2}\right), P\left(S_{1}\right) \cup P\left(S_{2}\right)\right)$ correspond to colorings $O\left(S_{1}\right) \cup O\left(S_{2}\right) \rightarrow \mathcal{C}$, and the elementary matrices in $\hat{A}_{d}\left(S_{1}, S_{2}\right)$ also correspond to such colorings. This gives an isometry between the euclidean spaces $T\left(O\left(S_{1}\right) \cup O\left(S_{2}\right), P\left(S_{1}\right) \cup P\left(S_{2}\right)\right)$ and $\hat{A}_{d}\left(S_{1}, S_{2}\right)$. By abusing the notation, we will identify the these spaces. Now the tensor construction defines maps $m: \hat{A}_{d}\left(S_{1}, S_{2}\right) \rightarrow P_{d}$ for all $S_{1}, S_{2} \in \mathcal{S}$. These maps have a unique common linear extension $m: \hat{A}_{d} \rightarrow P_{d}$.

Let us observe that

$$
T\left(O\left(S_{1}\right) \cup O\left(S_{2}\right), P\left(S_{1}\right) \cup P\left(S_{2}\right)\right)=T\left(O\left(S_{1}\right), P\left(S_{1}\right)\right) \otimes T\left(O\left(S_{2}\right), P\left(S_{2}\right)\right)
$$


and that for $v_{1} \in T\left(O\left(S_{1}\right), P\left(S_{2}\right)\right), v_{2} \in T\left(O\left(S_{2}\right), P\left(S_{2}\right)\right)$ we have that

$$
m\left(v_{1}\right) m\left(v_{2}\right)=m\left(v_{1} \otimes v_{2}\right) .
$$

Let

Is is clear that

$$
\hat{B}_{d, m}=\bigoplus_{S \in \mathcal{S}, \mu(S) \leq m} T(O(S), P(S))
$$

$$
\hat{B}_{d, m} \otimes \hat{B}_{d, m}=\hat{A}_{d, m}
$$

and that

$$
m\left(v_{1} \otimes v_{2}\right)=m\left(v_{1}\right) m\left(v_{2}\right)
$$

for an arbitrary pair $v_{1}, v_{2} \in \hat{B}_{d, m}$.

4.6. The averaging operator. We define the averaging operator $\xi: P_{d} \rightarrow I_{d}$ by

$$
\xi(g)=\int_{\alpha \in O_{d}(\mathbb{R})} g^{\alpha} d \nu
$$

where $\nu$ is the normalized Haar measure on the orthogonal group $O_{d}(\mathbb{R})$.

Lemma 4.8. The following diagram is commutative:

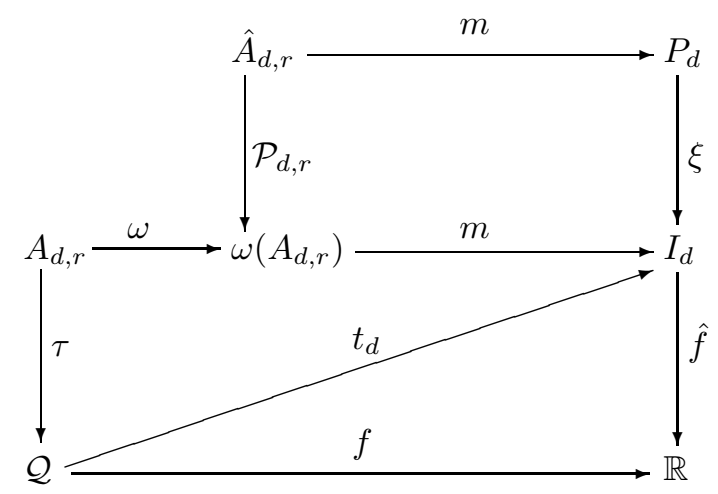

Proof. Note that each map on the diagram is $\mathbb{R}$-linear, and so it is enough to check the commutativity for an appropriately chosen generating system of the spaces.

First we prove that $\xi \circ m=m \circ \mathcal{P}_{d, m}$ by checking it for the spaces $\hat{A}_{d}\left(S_{1}, S_{2}\right)$. Recall that $\hat{A}_{d}\left(S_{1}, S_{2}\right)$ is identified with the euclidean space

$$
T=T\left(O\left(S_{1}\right) \cup O\left(S_{2}\right), P\left(S_{1}\right) \cup P\left(S_{2}\right)\right)
$$

and that the orthogonal group $O_{d}(\mathbb{R})$ is acting on $T$ by taking the tensor product of the actions on $V$. This action commutes with the map $m$ and so we have that

$$
m\left(\int_{\alpha \in O_{d}(\mathbb{R})} t^{\alpha} d \nu\right)=\xi(m(t))
$$

for all $t \in T$. Since the action of $O_{d}(\mathbb{R})$ preserves the scalar product on $T$ one gets that $\int_{\alpha \in O_{d}(\mathbb{R})} t^{\alpha} d \nu$ is the orthogonal projection of $t$ to the space of invariant elements. Therefore it suffices to prove that $\omega\left(A_{d, r}\right)$ is the space of invariants. This follows from Weyl's first fundamental theorem as described in section 4.3 . 
One gets $m \circ \omega=t_{d} \circ \tau$ by showing that

$$
m\left(\omega\left(a\left(S_{1}, S_{2}, M\right)\right)\right)=t_{d}\left(\tau\left(a\left(S_{1}, S_{2}, M\right)\right)\right)
$$

for all triples $S_{1}, S_{2}, M$. This follows immediately from the definitions.

The statement $\hat{f} \circ t_{d}=f$ is proved in corollary 4.4.

Lemma 4.9. If $g \in P_{d}$, then for a sufficiently large natural number $r$ there is a symmetric positive semi-definite matrix $M$ in $\hat{A}_{d, r}$ such that $m(M)=g^{2}$.

Proof. If $r$ is a large enough natural number, then

$$
g=\sum_{S \in \mathcal{S}, \mu(S) \leq r} g_{S}
$$

where $g_{S}$ is an element of $W_{S}$. Let us represent each $g_{S}$ by an element $t_{S}$ in the space $T(O(S), P(S))$ such that $m\left(t_{S}\right)=g_{S}$. This is possible because $m$ is a surjective map to $W_{S}$. Setting

$$
t=\sum_{S \in \mathcal{S}, \mu(S) \leq m} t_{S} \in \hat{B}_{d, m}
$$

we have that $m(t)=g$ and that $m(t \otimes t)=g^{2}$. On the other hand $t \otimes t$ is represented as a rank 1 positive semi-definite matrix $M$ in $\hat{A}_{d, r}$.

Lemma 4.10. If $g \in P_{d}$, then $\hat{f}\left(\xi\left(g^{2}\right)\right) \geq 0$.

Proof. Using lemma 4.9 we get that for a sufficiently large $r$ there is a symmetric positive semi-definite matrix $M \in \hat{A}_{d, r}$ such that $m(M)=g^{2}$. From lemma 4.6 we obtain that $\mathcal{P}_{d, r}(M)=K^{2}$ where $K$ is a symmetric matrix from $\omega\left(A_{d, r}\right)$. Let $\bar{K}$ be a preimage of $K$ under the map $\omega$. We have that $\omega\left(\bar{K} \bar{K}^{T}\right)=K^{2}$. By lemma 4.7 it follows that $f\left(\tau\left(\bar{K} \bar{K}^{T}\right)\right) \geq 0$. Lemma 4.8 implies that

$$
f\left(\tau\left(\bar{K} \bar{K}^{T}\right)\right)=\hat{f}\left(m\left(\omega\left(\bar{K} \bar{K}^{T}\right)\right)\right)=\hat{f}\left(m\left(K^{2}\right)\right) .
$$

It follows that $\hat{f}\left(m\left(\mathcal{P}_{d, r}(M)\right)\right) \geq 0$. Using lemma 4.8 again we obtain that

$$
\hat{f}(\xi(m(M))) \geq 0,
$$

which completes the proof.

4.7. Extension of $\hat{f}$ to $P_{d}$. In this section we finish the proof of our main theorem by showing that $\hat{f}: I_{d} \rightarrow \mathbb{R}$ extends to a homomorphism $\bar{f}: P_{d} \rightarrow \mathbb{R}$. This is clearly enough because the edge coloring model defined by

$$
t(v)=\bar{f}\left(x_{v}\right), v \in \mathbb{N}^{d},
$$

is a real valued edge coloring model which represents the graph parameter $f$. We will need the following well known consequence of the so-called Positivestellensatz (see: [2]).

Theorem 4.11. Let $g \in \mathbb{R}\left[x_{1}, x_{2}, \ldots, x_{n}\right]$ be a polynomial such that it has no root in $\mathbb{R}^{n}$. Then there exist polynomials $p, f_{1}, f_{2}, \ldots, f_{h}$ for some natural number $h$ such that

$$
p g=1+f_{1}^{2}+f_{2}^{2}+\cdots+f_{h}^{2} .
$$


Let $P_{d, r}$ be the subring of $P_{d}$ which is generated by the variables $\left\{x_{v} \mid h(v) \leq\right.$ $\left.r, v \in \mathbb{N}^{d}\right\}$. Since $P_{d, r}$ is the direct sum of the spaces $W_{S}$ where $S$ is a multiset of $\{0,1, \ldots, r\}$ we have that $P_{d, r}$ is invariant under the action of $O_{d}(\mathbb{R})$. Lemma 4.5 shows that $I_{d, r}=I_{d} \cap P_{d, r}$ is the set of invariant polynomials in $P_{d, r}$. Let $N_{d, r}$ be the kernel of the homomorphism $\hat{f}: I_{d, r} \rightarrow \mathbb{R}$.

Lemma 4.12. The homomorphism $\hat{f}: I_{d, r} \rightarrow \mathbb{R}$ extends to a homomorphism $\bar{f}_{r}: P_{d, r} \rightarrow \mathbb{R}$.

Proof. First of all note that $P_{d, r}$ is a polynomial ring with $t_{r}=\sum_{i=0}^{r}\left(\begin{array}{c}i+d-1 \\ d-1\end{array}\right)$ variables. It suffices to prove that there is a point $x$ in $\mathbb{R}^{t_{r}}$ which is a common root for all the polynomials in $N_{d, r}$ because the substitution of $x$ into polynomials from $P_{d}$ would yield a homomorphism of the required form. Let $M$ be the ideal generated by $N_{d, r}$ in $P_{d, r}$. Since $P_{d, r}$ is Noetherian we have that there are finitely many polynomials $g_{1}, g_{2}, \ldots, g_{k} \in N_{d, r}$ which generate $M$ as an ideal. We prove by contradiction that $g_{1}, g_{1}, \ldots, g_{k}$ have a common root. Assume that it is not true. Then $s=\sum_{i=1}^{k} g_{i}^{2}$ is a polynomial in $N_{d, r}$ which is positive everywhere in $\mathbb{R}^{t_{r}}$. Using theorem 4.11 we get that there is a polynomial $p \in P_{d, r}$ such that

$$
p s=1+f_{1}^{2}+f_{2}^{2}+\cdots+f_{h}^{2}
$$

for some natural number $h$. Applying the averaging operator $\xi$ for both sides we get that

$$
\xi(p) s=1+\xi\left(f_{1}^{2}\right)+\xi\left(f_{2}^{2}\right)+\cdots+\xi\left(f_{h}^{2}\right)
$$

because $s$ is invariant under the action of $O_{d}(\mathbb{R})$. The left side is an element of $N_{d, r}$ since $N_{d, r}$ is an ideal in $I_{d, r}$ and $\xi(p)$ is an element of $I_{d, r}$. This is a contradiction because lemma 4.10 shows that

$$
\hat{f}\left(1+\xi\left(f_{1}^{2}\right)+\xi\left(f_{2}\right)^{2}+\cdots+\xi\left(f_{h}^{2}\right)\right) \geq 1
$$

which means that the right side in not an element of $N_{d, r}$.

Lemma 4.13. The map $\hat{f}: I_{d} \rightarrow \mathbb{R}$ extends to a homomorphism $\bar{f}: P_{d} \rightarrow \mathbb{R}$.

Proof. Let

$$
g_{s}=\sum_{h(v)=s, v \in \mathbb{N}^{d}} g_{v}^{2} .
$$

It is easy to see that $g_{s}=t_{d}\left(G_{s}\right)$ where $G_{s}$ is the graph with two nodes which are connected by $s$ edges. This implies that $g_{s}$ is an element of $I_{d}$. Let $\bar{f}_{r}$ be a map described by lemma 4.12. If $r \geq s$, then $g_{s}$ is in $P_{d, r}$ and

$$
f\left(G_{s}\right)=\hat{f}\left(g_{s}\right)=\sum_{h(v)=s, v \in \mathbb{N}^{d}} \bar{f}_{r}\left(g_{v}\right)^{2} .
$$

It follows that $\left|\bar{f}_{r}\left(g_{v}\right)\right| \leq \sqrt{f\left(G_{s}\right)}$ for all $v \in \mathbb{N}^{d}$. Using these inequalities we have that there is an infinite sequence $r_{1}<r_{2}<\ldots$ of natural numbers such that $\hat{f}_{r_{i}}\left(x_{v}\right)$ is convergent for all fixed vector $v \in \mathbb{N}^{d}$. This means that $\hat{f}_{r_{i}}(p)$ is convergent for all polynomial $p \in P_{d}$ and the limit is a homomorphism which is an extension of $\hat{f}$ to $P_{d}$. 


\section{ACKNOWLEDGEMENTS}

I am very indebted to László Lovász for introducing me to this beautiful subject, and for the many exciting discussions without which this paper couldn't have been written. I am also indebted to Michael H. Freedman and Mátyás Domokos for their kind help and suggestions.

\section{REFERENCES}

[1] M. Atiyah: The geometry and physics of knots, Cambridge University Press, Cambridge, 1990. MR:1078014 (92b:57008)

[2] J. Bochnak, M. Coste, M. Roy: Real algebraic geometry, Springer-Verlag, Berlin, 1998. MR1659509 (2000a:14067)

[3] M. H. Freedman, A. Kitaev, C. Nayak, J. Slingerland, K. Walker, Zhenghan Wang: Universal manifold pairings and positivity, Geom. Topol. 9 (2005), 2303-2317. MR 2209373 (2006k:57080)

[4] M. H. Freedman, L. Lovász, A. Schrijver: Reflection positivity, rank connectivity, and homomorphism of graphs, J. Amer. Math. Soc. 20 (2007), 37-51. MR2257396

[5] Personal communication with M.H. Freedman, L. Lovász, A. Schrijver.

[6] L. Lovász: The rank of connection matrices and the dimension of graph algebras, European J. Combin. 27 (2006), 962-970. MR2226430(2007e:05101)

[7] L. Lovász and B. Szegedy: Limits of dense graph sequences, J. Combin. Theory Ser. B 96 (2006), 933-957. MR2274085

[8] H. Weyl: The Classical Groups, their Invariants and Representations, Princeton Mathematical Series, vol. 1, Princeton Univ. Press, Princeton, 1946. MR.1488158 (98k:01049)

Microsoft Research, One Microsoft Way, Redmond, Washington 98052 